\title{
Sleep technologist performance: a call for standardization and performance feedback
}

\author{
Reena Mehra
}

Received: 13 May 2009/Accepted: 15 May 2009 / Published online: 30 June 2009

(C) Springer-Verlag 2009

In their article in Sleep and Breathing, Surani and colleagues describe a sleep laboratory questionnaire implemented as a quality assurance instrument to provide standardized feedback to the sleep lab technologist. The authors accurately identify the lack of recognized standardized mechanisms to provide feedback to the sleep technologist with the goal of improving upon the quality of care being provided in the sleep laboratory setting. This is an area of importance, in particular, given the burgeoning growth in the number of sleep centers and therefore attendant sleep studies being performed. There are currently no standardized means of assessing the technologist-related performance of sleep study data acquisition that has been put forth by the American Academy of Sleep Medicine. By the same token, global technologist performance quality measures are not formally incorporated or mandated in the sleep laboratory accreditation process.

The authors employed a pre- and post-intervention scheme to assess the effect of the implementation of a technologist performance evaluation which encompasses the following domains: patient assessment/education, signal quality, nocturnal polysomnogram orders, chart organization, non-compliant patient, arrhythmia documentation,

\section{R. Mehra}

Division of Pulmonary, Critical Care, and Sleep Medicine,

Department of Medicine,

University Hospitals Case Medical Center,

Cleveland, $\mathrm{OH}$, USA

\section{R. Mehra $(\triangle)$}

Center of Clinical, Investigation,

Department of General Medical Sciences,

Case School of Medicine,

2103 Cornell Road, Room 6126,

Cleveland, OH 44106, USA

e-mail: reena.mehra@case.edu equipment malfunction, positive airway pressure titration quality, questionnaire administration, scoring of sleep staging, and overall documentation. A random review of 100 charts pre-implementation and 1,736 charts postimplementation was performed. The authors showed that the score on this quality improvement tool $(0-100)$ increased from $75+/-4.12$ to $87.53+/-0.91(p=0.0001)$ [3].

There are several important points that this work raises. The first is the content of the constructs used to assess the technologist performance. Key technologist performance determinants which one may posit should be considered for inclusion are those that affect and impact patient care and those that affect study quality. Patient care-related components that may be considered include compliance with the sleep laboratory emergency protocol/procedures with appropriate contact of the sleep physician and performance of positive airway pressure acclimatization, mask fitting, and desensitization. The latter is particularly of importance given data demonstrating that early adherence predicts later adherence [1]. Study quality characteristics including effective troubleshooting of equipment malfunction and lab equipment maintenance are relevant given the impact that suboptimal procedures may result in difficulty with scoring and interpretation of the collected physiologic sleep data which may lead to misdiagnosis or potentially the need for a repeat sleep study. For example, our sleep lab utilizes an instrument which incorporates the following primary content areas of performance: completion of paperwork, adequacy of electrode applications including biocalibrations, data acquisition (monitoring and quality), adherence to procedures (patient preparation, patient care including verification of the medical order, pre-sleep study data collection such as height and weight, adherence to protocols, and adequacy of positive airway pressure titrations), adequacy of documentation, and lab equipment mainte- 
nance. In our laboratory, monthly quality percentage scores are calculated and reviewed with the technologists to provide feedback.

With respect to the quality of the proposed sleep technologist performance model, features of reliability and validity should be ascertained. For example, are these measures of technologist performance generalizable; i.e. consistent and reliable assessments that can be obtained in any lab setting? The individual who assesses these performance characteristics should also ideally be consistent, and should demonstrate adequate intra-individual reliability. If another assessor is identified, then the interindividual reliability of the rating of these performance characteristics should be intact. Although this tool may be a useful quality assessment tool, the ability of this tool to result in technologist quality improvement may be difficult to discern given extraneous issues at hand. Although the authors do note improvement in the technologist performance with the use of this tool; other relevant temporal factors such as changes in lab directorship, location, lab procedures, and personal difficulties that the technologist encounters should also be accounted for as these factors may affect technologist performance. In addition, the role of the "Hawthorne effect" [2] cannot be excluded as the improvement in technologist performance that was noted may have been a result of increased awareness of being assessed by this quality instrument rather than a result of the specific feedback obtained from or particular aspects of the tool itself.

In summary, the authors should be commended for highlighting the importance of sleep laboratory technologist performance feedback, and developing a quality assurance and potentially a quality improvement tool that will possibly have the ability to enhance the quality of sleep studies leading to optimizing overall patient care in addition to facilitating the diagnosis and even management of patients with sleep disorders. Future areas of investigation in this realm should include careful consideration of the content of the technologist performance constructs as well as ensuring generalizability across various sleep labs to allow for uniform standardization.

\section{References}

1. Budhiraja R, Parthasarathy S, Drake CL, Roth T, Sharief I, Budhiraja P, Saunders V, Hudgel DW (2007) Early CPAP use identifies subsequent adherence to CPAP therapy. Sleep 30(3):320324

2. McCarney R, Warner J, Iliffe S, van Haselen R, Griffin M, Fisher P (2007) The Hawthorne effect: a randomised, controlled trial. BMC Med Res Methodol 7:30

3. Surani S, Aguillar R, Aguillar R, Subramanian S (2009) Standardization of quality assurance for sleep technologist: a model. Sleep Breath doi:10.1007/s11325-009-0271-5 